Mineralogical Journal, Vol. 5, No. 4, Pp. 285-290, Mar., 1968

\title{
THE OCCURRENCE OF TEINEITE FROM THE KAWAZU (RENDAIJI) MINE, SHIZUOKA PREFECTURE, JAPAN
}

\author{
AKIRA KATO \\ Division of Mineralogy, Smithsonian Institution, U.S. National Museum, \\ Washington, D.C. U.S.A. \\ and \\ KIN-ICHI SAKURAI \\ Department of Geology, National Science Museum, \\ Ueno, Tokyo, Japan
}

\begin{abstract}
Teineite from the new locality, Kawazu mine, Shizuoka Prefecture, Japan, is described and its relation to an associating but unidentified copper-tellurium sulphide is discussed.

Teineite, $\mathrm{CuTeO}_{3} \cdot 2 \mathrm{H}_{2} \mathrm{O}$, a rare secondary mineral only known from the original locality, Teine mine, Hokkaido (Yoshimura (1936)), was found from the Kawazu (Rendaiji) mine, Shizuoka prefecture, Japan.

The ore deposits of the Kawazu (Rendaiji) mine consist of epithermal veins cutting altered Neogene pyroclastic rocks (Kato, Shibata and Nakamoto (1933)), and had been worked for gold and silver till 1963. The veins are classified into two kinds according to the nature of ores, that is, one gold-silver-tellurium-bearing quartz vein and some gold-silver-copper-zinc-lead-bearing quartz veins with a very small amount of tellurium in which the studied material was found. From the former, the occurrences of tellurium (Watanabe (1936)), rickardite
\end{abstract}


(Watanabe (1934)), hessite, enpressite and stuetzite (Takasu (1965)), tellurite (Kato, Shibata and Nakamoto (1933)), paratellurite (Sakurai and Kato (1965a)) and spiroffite (Sakurai and Kato (1965b)) are known, and those of chalcopyrite, sphalerite, galena, electrum, argentite and tetrahedrite (Kato, Shibata and Nakamoto (1933)), an unnamed coppertellurium-sulphide mineral (cubic, $a_{0}=10.26 \AA$, with tetrahedrite-type $\mathrm{X}$-ray powder pattern), inesite (Kato (1930)), and johannsenite (Sakurai and Kato (unpublished)) from the latter. The specimen studied was collected by the second author about thirty years ago. It is a very small piece of gray-black ore with drusy parts surrounded by minute rock crystals on which an azure-blue bundle of teineite up to about $1 \times 1 \times 2 \mathrm{~mm}$. in size is present. The gray black part consists of minute aggregate of pyrite, the above-mentioned copper-telluriumsulphide mineral and quartz. The drusy parts are locally rimmed by light green colour due to dissemination of very minute brochantite grains, which form in places tiny mammilary aggregates less than $0.5 \mathrm{~mm}$. in diameter. The teineite bundle is isolated from these aggregates and composed of aggregates of long to short prismatic crystals with rugged faces. Part of the prisms is superficially covered with a blue green undeterminable alteration product.

Since the amount of specimen available for the determination was very small, the $X$-ray powder and fluorescent studies were made. The X-ray powder data are virtually identical with those by Mandarino (1964), and Uetani, Kato and Imayoshi (1965), except a few unindexed diffraction lines $(d=4.53,3.81$ and $1.547 \AA$ ) probably due to the contaminative alteration product, which could not be identified by the A.S.T.M. Card File (Table 1). From the indexing, the unit cell constants are calculated as $a_{0}=6.63_{1} \AA, b_{0}=9.60_{1} \AA, c_{0}=7.43_{0} \AA$. The $\mathrm{X}$-ray fluorescent analysis gave the presence of copper and tellurium as the major constituents.

It is worth mentioning that teineite from the original locality is found in druses of tellurium-barite ore virtually void of significant 
Table 1. X-ray powder data for teineites from the Teine mine, Hokkaido and the Kawazu (Rendaiji) mine, Shizuoka prefecture, Japan

\begin{tabular}{|c|c|c|c|c|c|c|}
\hline \multicolumn{2}{|c|}{1.} & \multicolumn{5}{|c|}{2.} \\
\hline$d(\AA)$ & $I$ & $d(\AA)$ & $I$ & Qobs. & $Q_{c a l c}$ & $h k l$ \\
\hline 5.90 & 25 & 5.89 & 30 & 0.030 & 0.029 & 011 \\
\hline 5.47 & 100 & 5.43 & 100 & 0.034 & 0.034 & 110 \\
\hline 4. 96 & 45 & 4.95 & 30 & 0.041 & 0.041 & 101 \\
\hline 4.77 & 2 & 4. 53 & 5 & & & 020 \\
\hline 4. 41 & 15 & 4. 40 & 20 & 0.052 & 0.052 & 111 \\
\hline 4.04 & 2 & 4.04 & 5 & 0.061 & 0.062 & 021 \\
\hline 3.89 & 10 & $\begin{array}{l}3.89 \\
3.81\end{array}$ & $\begin{array}{r}10 \\
5\end{array}$ & 0.066 & 0.066 & 120 \\
\hline 3.72 & 5 & 3.70 & 10 & 0.073 & 0.073 & 002 \\
\hline 3.45 & 100 & 3.44 & 100 & 0.085 & $\begin{array}{l}0.083 \\
0.084\end{array}$ & $\begin{array}{l}012 \\
121\end{array}$ \\
\hline 3.32 & 10 & 3.31 & 5 & 0.091 & 0.091 & 200 \\
\hline 3.24 & 5 & 3.24 & 5 & 0.095 & 0.095 & 102 \\
\hline 3.14 & 10 & 3.13 & 10 & 0.102 & 0.102 & 210 \\
\hline 3.07 & 55 & 3.07 & 60 & 0.106 & 0.106 & 112 \\
\hline 2.940 & 25 & 2.94 & 60 & 0.116 & $\begin{array}{l}0.116 \\
0.116\end{array}$ & $\begin{array}{l}031 \\
022\end{array}$ \\
\hline 2.890 & 50 & 2.89 & 60 & 0.120 & $\begin{array}{l}0.120 \\
0.121\end{array}$ & $\begin{array}{l}211 \\
130\end{array}$ \\
\hline 2.730 & 10 & 2.73 & 10 & 0.134 & 0.135 & 220 \\
\hline 2.687 & 20 & 2.68 & 20 & 0.139 & $\begin{array}{l}0.139 \\
0.139\end{array}$ & $\begin{array}{l}131 \\
122\end{array}$ \\
\hline 2.564 & 30 & 2.56 & 20 & 0.153 & 0.153 & 221 \\
\hline 2.477 & 15 & 2.47 & 10 & 0.164 & 0.164 & 202 \\
\hline 2. 400 & 35 & 2.40 & 40 & 0.174 & $\begin{array}{l}0.174 \\
0.174\end{array}$ & $\begin{array}{l}040 \\
013\end{array}$ \\
\hline 2.279 & 2 & 2.28 & 10 & 0.193 & $\begin{array}{l}0.192 \\
0.193\end{array}$ & $\begin{array}{l}041 \\
132\end{array}$ \\
\hline 2.259 & 1 & 2.26 & 10 & 0.196 & $\begin{array}{l}0.195 \\
0.197\end{array}$ & $\begin{array}{l}140 \\
113\end{array}$ \\
\hline 2.204 & 15 & 2.20 & 20 & 0.207 & 0.206 & 023 \\
\hline
\end{tabular}




\begin{tabular}{|c|c|c|c|c|c|c|}
\hline & & & & & 0.207 & 231 \\
\hline & & & & & 0.207 & 222 \\
\hline 2.158 & 10 & 2.16 & 10 & 0.214 & & 141 \\
\hline & & & & & 0.216 & 310 \\
\hline 2.125 & 2 & & & & & 301 \\
\hline 2.091 & 20 & 2.09 & 30 & 0.229 & 0.229 & 123 \\
\hline 2.074 & 5 & 2.07 & 10 & 0.233 & 0.234 & 311 \\
\hline 2.013 & 25 & 2.01 & 20 & 0.248 & 0.246 & 042 \\
\hline & & & & & 0.249 & 320 \\
\hline & & 1. 984 & 5 & 0.2541 & 0.2542 & 203 \\
\hline 1. 960 & 10 & 1.959 & 10 & 0.2606 & 0.2607 & 033 \\
\hline & & & & & 0.2614 & 232 \\
\hline 1.943 & 1 & 1.940 & 10 & 0.2657 & 0.2648 & 240 \\
\hline & & & & & 0.2650 & 213 \\
\hline & & & & & 0.2667 & 321 \\
\hline 1.884 & 5 & 1.881 & 10 & 0.2826 & 0.2829 & 241 \\
\hline & & & & & 0.2835 & 133 \\
\hline 1.865 & 5 & 1.862 & 10 & 0.2884 & 0.2886 & 312 \\
\hline & & & & & 0.2894 & 051 \\
\hline 1.858 & 5 & & & & 0.2898 & 004 \\
\hline & & 1.844 & 5 & 0.2817 & 0.2829 & 241 \\
\hline 1.823 & 2 & 1.818 & 10 & 0.3026 & 0.3029 & 330 \\
\hline 1.795 & 5 & 1. 789 & 5 & 0.3125 & 0.3122 & 151 \\
\hline & & & & & 0.3126 & 104 \\
\hline 1. 758 & 5 & 1.760 & 10 & 0.3228 & 0.3235 & 114 \\
\hline 1.725 & 15 & 1.723 & 20 & 0.3368 & 0.3366 & 043 \\
\hline & & & & & 0.3373 & 242 \\
\hline 1. 688 & 5 & 1.688 & 10 & 0.3510 & 0.3519 & 233 \\
\hline 1.669 & 5 & 1.670 & 5 & 0.3586 & 0.3594 & 143 \\
\hline 1. 660 & 5 & & & & 0.3648 & 400 \\
\hline 1.652 & 10 & 1. 622 & 10 & 0.3801 & 0.3788 & 340 \\
\hline & & & & & 0.3791 & 313 \\
\hline & & & & & 0.3810 & 204 \\
\hline 1.601 & 2 & 1.600 & 5 & 0.3906 & 0.3906 & 060 \\
\hline & & & & & 0.3919 & 214 \\
\hline 1.561 & 2 & 1. 561 & 10 & 0.4104 & 0.4103 & 134 \\
\hline & & & & & 0.4116 & 323 \\
\hline
\end{tabular}




\begin{tabular}{|c|c|c|c|c|c|c|}
\hline \multirow{4}{*}{1.531} & \multirow{4}{*}{5} & 1.547 & 5 & \multirow{3}{*}{0.4268} & & \\
\hline & & \multirow[t]{2}{*}{1.531} & \multirow[t]{2}{*}{10} & & 0.4273 & 421 \\
\hline & & & & & 0.4278 & 243 \\
\hline & & 1.512 & $10 \mathrm{~b}$ & 0.4374 & 0.4373 & 402 \\
\hline \multirow[t]{2}{*}{ 1. 499} & \multirow[t]{2}{*}{2} & \multirow[t]{2}{*}{1.492} & \multirow[t]{2}{*}{5} & \multirow[t]{2}{*}{0.4492} & 0.4482 & 412 \\
\hline & & & & & 0.4498 & 071 \\
\hline \multirow[t]{3}{*}{1.470} & \multirow[t]{3}{*}{5} & \multirow[t]{3}{*}{ 1. 469} & \multirow[t]{3}{*}{10} & \multirow[t]{3}{*}{0.4634} & 0.4631 & 062 \\
\hline & & & & & 0.4634 & 044 \\
\hline & & & & & 0.4637 & 015 \\
\hline \multirow[t]{4}{*}{1.447} & \multirow[t]{4}{*}{5} & 1. 446 & 10 & 0.4783 & 0.4787 & 234 \\
\hline & & 1. 431 & 5 & 0.4883 & 0.4865 & 115 \\
\hline & & 1.423 & 5 & 0.4939 & 0.4946 & 351 \\
\hline & & 1.389 & 5 & 0.5183 & 0.5190 & 125 \\
\hline 1.367 & 10 & 1.365 & 10 & 0.5367 & 0.5384 & 440 \\
\hline \multirow[t]{4}{*}{ 1. 344} & \multirow[t]{4}{*}{5} & \multirow[t]{4}{*}{ 1. 343} & \multirow[t]{4}{*}{10} & \multirow[t]{4}{*}{0.5544} & 0.5536 & 063 \\
\hline & & & & & 0.5543 & 262 \\
\hline & & & & & 0.5547 & 244 \\
\hline & & & & & 0.5550 & 215 \\
\hline \multicolumn{3}{|c|}{$a_{0}=6.639 \AA$} & \multicolumn{4}{|c|}{$a_{0}=6.63_{1} \pm 0.005 \AA$} \\
\hline \multicolumn{3}{|c|}{$b_{0}=9.606 \AA$} & \multicolumn{4}{|c|}{$b_{0}=9.60_{1} \pm 0.005 \AA$} \\
\hline \multicolumn{2}{|c|}{$c_{0}=7.432 \AA$} & & \multicolumn{3}{|c|}{$c_{0}=7.43_{0} \pm 0.005 \AA$} & \\
\hline
\end{tabular}

1. Teineite. Teine mine, Hokkaido, Japan. $\mathrm{Cu} / \mathrm{Ni}$ radiation. Intensities were measured on diffractometer scale. After Uetani, Kato and Imayoshi (1965).

2. Teineite. Kawazu mine, Shizuoka prefecture, Japan. Cu/Ni radiation. Camera diam. $114.59 \mathrm{~mm}$. Intensities were estimated visually. $b=$ broad.

copper mineral. Hence, the copper is considered to have been derived from other sources somewhat distant from the site of its formation. While for the present specimen, the presence of a copper-tellurium mineral close to it suggests itself to be the direct source of copper and tellurium provided that this teineite is of secondary origin.

Though we have not succeeded to accumulate much material to explain the rarity of this mineral, the present study suggests that the copper-tellurium-sulphide mineral may be the direct source of 
this teineite under such an appropriate condition as copper behaves as divalent copper ion and tellurium takes the form of $\mathrm{TeO}_{3}{ }^{-2}$ ion.

This work was done while one of the authors (A.K.) was at the Division of Mineralogy, Smithsonian Institution, U.S. National Museum as a Postdoctoral Research Associate sponsored by the National Research Council, U. S. A.

\section{REFERENCES}

Kato, T. (1930). J. Geol. Soc. Japan. 37, 35-38 (in Japanese).

Kato, T., Shibata, H. \& Nakamoto, A. (1933). J. Geol. Soc. Japan, 40, 233241 (in Japanese).

Mandarino, J.A. (1964). Amer. Min., 49, 1461-1485.

Sakural, K. \& Kato, A. (1965a). J. Miner. Soc. Japan, 7, 346-348 (in Japanese).

Sakurai, K. \& Kato, A. (1965b). J. Miner. Soc. Japan, 7, 348-350 (in Japanese).

Takasu, S. (1965). J. Miner. Soc. Japan, 7, 350-355 (in Japanese).

Uetani, K., Kato, A. \& Imayoshi, T. (1965). Bull. Nat. Sci. Mus. Tokyo, 8, 95-97.

Watanabe, M. (1934). J. Jap. Assoc. Miner. Petro. Econ. Geol., 12, 89-96 (in Japanese).

Watanabe, T. (1936). J. Fac. Sci. Hokkaido Univ. Ser. IV, 3, 101-111.

Yoshimura, T. (1936). J. Jap. Assoc. Miner. Petro. Econ. Geol., 16, 225-234 (in Japanese).

Manuscript received 13 December 1967. 